\title{
Nonmetric Lens Distortion Calibration: Closed-form Solutions, Robust Estimation and Model Selection *
}

\author{
Moumen T. El-Melegy \\ Electrical Engineering. Dept. \\ Assiut University \\ Assiut, Egypt \\ mmelegy@aun.edu.eg
}

\author{
Aly A. Farag \\ Computer Vision and Image Processing Lab. \\ University of Louisville \\ Louisville, KY 40292 \\ farag@cvip.louisville.edu
}

\begin{abstract}
This paper addresses the problem of calibrating camera lens distortion, which can be signifcant in medium to wide angle lenses. While almost all existing nonmetric distortion calibration methods need user involvement in one form or another, we present an automatic approach based on the robust the-least-median-of-squares (LMedS) estimator. Our approach is thus less sensitive to erroneous input data such as image curves that are mistakenly considered as projections of $3 D$ linear segments. Our approach uniquely uses fast, closed-form solutions to the distortion coeffcients, which serve as an initial point for a non-linear optimization algorithm to straighten imaged lines. Moreover we propose a method for distortion model selection based on geometrical inference. Successful experiments to evaluate the performance of this approach on synthetic and real data are reported.
\end{abstract}

\section{Introduction}

Camera lens distortion can be signifcant in medium to wide angle lenses. The distortion parameters are often calibrated along with all (extrinsic and intrinsic) parameters of the camera model [13]. The problem with these methods is their need for metric information about the imaged scene. Moreover, there is some kind of coupling between internal parameters, including distortion parameters, and external parameters that result in high errors on the camera internal parameters [5]. In contrast, another family of non-metric methods have been proposed, which do not rely on known scene points $[4,5,8,11]$. Instead, most these methods rely on the fact that straight lines in the scene must always perspectively project to straight lines in the image.

* This work was supported in part by the US Army Grants DABT6001-P-0046 and DABT60-02-P-0063.
In this paper, we propose a complete, automatic nonmetric approach to camera lens calibration. Several aspects about our approach are novel. Firstly, in almost all existing nonmetric distortion calibration methods $[4,8,11]$, some user involvement for data preparation is needed in one form or another. For example, the user should manually select the image curves that correspond to scene linear segments [11]. We propose to use a robust approach based on the least median of squares (LMedS) estimator to discard outliers in the data. Our approach is thus able to proceed in a fullyautomatic manner while being less sensitive to erroneous data such as image curves that are mistakenly considered as projections of 3D linear segments.

Secondly, we £nd fast, closed-form solutions to the distortion coeffcients, which are refned later using non-linear search techniques to $£$ nd the best distortion parameters that straighten the image lines. The existing methods typically start with a non-linear optimization algorithm assuming zero distortion, which may lead to a less accurate result and/or a longer search time with increased probability of being stuck in a local minimum.

Lastly, the paper addresses the problem of how to select a proper model for lens distortion. Almost all previous efforts (e.g., $[13,4,5,8,11])$ calibrated a £xed, pre-speci£ed distortion model. As such, they may suffer from over/underparameterization. Here we propose to exploit, for the £rst time, geometric inference $[6,7]$ for distortion model selection as it £ts more geometrical problems of computer vision. We investigate and compare the application of both the geometric AIC and geometric MDL criteria.

This paper is organized as follows. Section II describes the camera distortion model. Section III presents our approach to distortion calibration, while a method for model selection is proposed in Section IV. Some experimental results are reported in Section V, followed by our concluding remarks in Section VI. 


\section{Camera Distortion Model}

The standard model for the radial and decentering distortion [13] is mapping from the distorted image coordinates, $\left(x_{d}, y_{d}\right)$, that are observable, to the undistorted image plan coordinates, $\left(x_{u}, y_{u}\right)$, which are not physically measurable, according to the equation:

$$
\begin{gathered}
x_{u}=x_{d}+\overline{x_{d}}\left(K_{1} r_{d}^{2}+K_{2} r_{d}^{4}+K_{3} r_{d}^{6}+\ldots\right)+ \\
{\left[P_{1}\left(r_{d}^{2}+2{\overline{x_{d}}}^{2}\right)+2 P_{2} \overline{x_{d} y_{d}}\right]\left[1+P_{3} r_{d}^{2}+\ldots\right]} \\
y_{u}=y_{d}+\overline{y_{d}}\left(K_{1} r_{d}^{2}+K_{2} r_{d}^{4}+K_{3} r_{d}^{6}+\ldots\right)+ \\
{\left[P_{2}\left(r_{d}^{2}+2{\overline{y_{d}}}^{2}\right)+2 P_{1} \overline{x_{d} y_{d}}\right]\left[1+P_{3} r_{d}^{2}+\ldots\right],}
\end{gathered}
$$

where

$$
\overline{x_{d}}=x_{d}-c_{x}, \overline{y_{d}}=y_{d}-c_{y}, \quad r_{d}^{2}={\overline{x_{d}}}^{2}+{\overline{y_{d}}}^{2},
$$

and $K_{1}, K_{2}, K_{3}$ are the coef£cients of radial distortion and $P_{1}, P_{2}$ and $P_{3}$ are the coef£cients of the decentering distortion. $r_{d}$ is the radius of an image point from the distortion center, defned as $\left(c_{x}, c_{y}\right)$ above. Typically, only few distortion parameters are modeled, as the higher order terms are comparatively insignifcant [13]. The lens distortion calibration problem thus becomes to recover the practically signifcant distortion coeffcients along with the distortion center $\left(c_{x}, c_{y}\right)$.

\section{Proposed Approach}

To £nd the distortion parameters, the following fact is often used: a camera follows the perspective camera model if and only if the projection of every $3 \mathrm{D}$ line in space onto the camera plane is a line. The techniques based on this fact lead to non-linear objective functions (error measures) that need ef£cient search algorithms in order to $£$ nd the best calibration parameters. One common such measure (denoted $\xi_{l}$ ) is the sum of squared distances of the edge points from the straight lines on which they should lie $[4,5,8]$.

In the following, we describe our proposed approach. At £rst, our approach, uniquely, derives closed-form solutions to the distortion coef£cients, which are re£ned by nonlinear search. Then we propose a robust version of the approach which follows the general outline [10] of the LMedS method after taking some critical issues into account.

\subsection{Closed-form Solutions}

Suppose we have a line $l$ in the undistorted image plane. Each point on the line is related to a point $\left(x_{d}, y_{d}\right)$ in the distorted image plane according to (1). It can be shown [2] that the slope of the line, $s$, in the undistorted plane is related to the slope of the tangent, $\frac{\delta y_{d}}{\delta x_{d}}$, to the curve at point $\left(x_{d}, y_{d}\right)$ by

$$
s\left(x_{d}, y_{d}\right)=\frac{\frac{\partial y_{u}}{\partial x_{d}}+\frac{\partial y_{u}}{\partial y_{d}} \frac{\delta y_{d}}{\delta x_{d}}}{\frac{\partial x_{u}}{\partial x_{d}}+\frac{\partial x_{u}}{\partial y_{d}} \frac{\delta y_{d}}{\delta x_{d}}},
$$

where all the four partial derivatives can be directly computed from (1). In the problem of distortion calibration, we usually have a number of distorted points in the image plane. Under the correct values of the distortion parameters, the slopes computed from the last equation for all these points should be the same if the points are to lie on the same line in the undistorted image. Therefore, we can defne the following distortion measure. Given a chain of edge points, $\left(x_{d}^{i}, y_{d}^{i}\right), i=1, \cdots, N$, that should belong to the same line in the undistorted image, we can compute approximately the slopes of the tangents at the chain points and hence we can solve for the distortion parameters that minimize the error $\xi_{s}=\sum_{i=2}^{N}\left(s\left(x_{d}^{i}, y_{d}^{i}\right)-s\left(x_{d}^{i-1}, y_{d}^{i-1}\right)\right)^{2}$. To improve the accuracy, several curves distributed through the image ought to be used with the error $\xi_{s}$ being computed for all the chains.

Closed-form solutions for rest of the distortion parameters can be obtained if the distortion center is assumed known (see below). At each point of the curves extracted from the distorted image, Eq. 2 can be applied, with the left-hand side (LHS) being the slope of the undistorted line to which these points belong. That slope can be estimated from the curve points by least-square linear regression ${ }^{1}$. With the LHS of Eq. 2 being known, each point will thus yield one linear equation in the distortion coeffcients. All the equations obtained from all points are stacked in the form $\mathbf{A} \mathbf{x}=\mathbf{b}$. This over-determined set of equations can be ef£ciently solved using singular value decomposition. The obtained solution is refned further using the LevenbergMarquardt (LM) algorithm minimizing the distortion error $\xi_{s}$ or $\xi_{l}$.

It was observed in our experiments as well as in [11, 4] that including both the distortion center and the decentering coeffcients in the non-linear search may lead to instability. Neither paper gave any explanation for this observation, while it was recommended in [11] the estimation of the distortion coef£cients be nested within a coarse-to-£ne search for the distortion center in order to avoid the instability. In [1], we are able to explain this observation, both analytically and experimentally, as both the distortion center and the decentering coeffcients tend to adjust for each other during the non-linear search. Thus by £xing the distortion center at a appropriate location (we use the image center) and then using the two decentering distortion coeffcients $P_{1}$ and $P_{2}$ to compensate for reasonable deviations of the center from

\footnotetext{
${ }^{1}$ Our experiments [1] verifed that the slope, unlike the intercept, obtained from best-£t line of the distorted curve points is usually close to the "unknown" slope of the undistorted line.
} 
the true location. As such, one can exclude the distortion center from the set of unknown variables. This reduces the dimension of the search space without signifcant loss in accuracy, which leads to faster calibration. Moreover, this result makes it even more straightforward to use the closedform solution.

\subsection{Robust Calibration}

The distortion calibration method requires a number of chains of edge points that correspond to real 3D linear segments. To meet this requirement, some user involvement in one form or another is needed. The user ought to select the edge chains that are projections of straight lines in the scene [11]. Moreover, some "sample" points from each selected chain can be picked out and fed to the calibration algorithm [11]. Besides, a number of system parameters, such as edge linking thresholds, may need manual tuning. A fully-automatic method should be more tolerant to erroneous data that might enter the estimation algorithm in different forms. For example, some image curves may be mistakenly considered as projections of 3D linear segments. Another error source is image curves that do really correspond to 3D linear segments but are linked together as one chain after the edge linking process. Using a smaller linking threshold can help but would yield smaller segments that may contain more noise than useful information about distortion. With a robust estimation method, one can risk using a bigger linking threshold to produce longer imaged segments that contain more useful information.

Outlying data will severely degrade the distortion estimation algorithm if one directly applies the methods described above or in the literature since they are all leastsquares techniques. We are aware of only one work [5] that tried to reduce the effect of outliers on distortion calibration. In that work, Devernay and Faugeras used a smaller linking threshold to produce the edge chains that are to be used by the optimization process. Then by repeating distortion minimization and polygonal approximation on the undistorted edges using the resultant distortion parameters, many outliers can be eliminated and longer, more useful segments can be obtained and thus more accurate calibration. Their technique relies on that undistorting the edges after the optimization would make identifying outliers during the next polygonal approximation easier. However this would not be the case when the image has severe distortion, when many 3D segments are broken into smaller edges, or when too many outliers are found in the data. In any of these cases, the distortion parameters obtained after the frst iteration will be highly perturbed, and will not make outlier identifcation in the next iteration any easier.

Here we propose an automatic method for lens distortion calibration based on robust estimators. The LMedS method estimates the parameters by solving the non-linear minimization problem: $\min \operatorname{median}_{i} r_{i}^{2}$, where $r_{i}$ denotes the residual of the $i^{\text {th }}$ datum. The algorithm which we have implemented for robustly estimating the lens distortion parameters generally follows the structure outlined in [10] and is summarized below. Some issues critical to the implementation and application of the LMedS method to the problem of distortion calibration are pointed out in the next subsection.

Given $n$ edge points, a Monte Carlo type technique is used to draw $m$ random subsamples of $q$ different points. For each subsample, indexed by $j$, we determine the distortion parameters $\mathcal{P}_{j}$ using the method described above. For each $\mathcal{P}_{j}$, we can determine the median of the squared residuals, denoted by $M_{j}$ with respect to the whole set of points. We retain the estimate $\mathcal{P}_{j}$ for which $M_{j}$ is minimal among all $m M_{j}$ 's. The number of subsamples $m$ should be big enough such that at least one of the subsamples is "good".

The LMedS effciency is poor in the presence of Gaussian noise [10]. To compensate for this de£ciency, one £rst make a good, robust estimate of the standard deviation of the errors of good data (inliers). This estimate is related to the median of the absolute values of the residuals, given by [10]: $\hat{\sigma}=1.4826[1+5 /(n-q)] \sqrt{M_{J}}$, where $M_{J}$ is the minimal median. Any data item whose error is larger than a certain number (e.g. $2.5-3.0$ ) of $\hat{\sigma}$ can be considered as an outlier and removed. The distortion parameters are Enally estimated by applying the distortion calibration algorithm once again on the inlying points.

\subsection{Implementation Details}

Here we discuss in more detail some issues related to the implementation of the robust algorithm. The frst issue is how to compute the residual $r_{i}$ for each point. The residual of a particular point should redect its own contribution to the ftting error of the model. Previously proposed distortion measures gauge the distortion carried by a point but in accordance with one or more points on the same imaged line, some of which may be outlying. Therefore once we solve for the distortion parameters for a subsample, the residual $r_{i}$ for each point is computed as the distance from the point to the line robustly estimated from the curve points. The bestft parameters of a line are computed by using the LMedS estimator once again. The robustly-estimated line slope is also used to fnd the closed-form solution.

Moreover, to select the points of a subsample, one should take care of two concerns. On one hand, the points of a subsample ought to be distributed across the image in order that the obtained parameters be not biased by the region from which the points come. On the other hand, the points selected should provide enough constraints to solve for the distortion parameters. For example, we need at least 
2 points from any line to impose one constraint on the distortion parameters. In order to consider these concerns and achieve higher effciency and stability, we used a random selection method based on bucketing techniques [14] on two levels: the frst to select a bucket from the image, and the second to select a line from the chosen bucket. The selection method works as follows.

The minimum and the maximum of the coordinates of the extracted edge points in the image are calculated. Then the region within these limits is divided into $b \times b$ buckets (we used $b=2$ ). Each extracted edge chain is attached to the bucket that includes most of the chain points. Buckets having no points are excluded. To generate a subsample of $q$ points, we randomly select $q / 2$ buckets, and then randomly choose a chain from each selected bucket. From each chosen chain, two points are picked out at random, one from the Erst half of the chain and another from the second half. Thus we end up with $q$ selected points per subsample. The required number of subsamples $m$ is determined based on the expected number of outliers in the data [10]. For more details, refer to [1].

Finally, an outline of the robust calibration algorithm is shown as Algorithm 1.

\section{Distortion Model Selection}

Algorithm 1 requires the distortion model be prespecifed through the model order $p$. This raises the problem of choosing the proper model for a given lens. The distortion model may be selected experimentally based on inspection of the input and after-distortion-correction images. The question here is how to select this model automatically and ef£ciently even in non-clear situations.

A naive idea is to choose from among candidate models the one that gives the smallest residual. This does not work, however, because a model with more degrees of freedom will be almost always chosen as it yields a smaller residual. For a fair comparison, one needs to compensate for the overft caused by excessive degrees of freedom. Model selection is one of the central subjects of statistical inference. Some of the widely adopted criteria for statistical model selection are Akaike's AIC [3] and Rissanen's MDL [9]. This problem has been generalized in abstract terms as geometric $£$ tting, for which a general theory of statistical optimization has been developed [6] so it becomes useful for geometric problems considered in computer vision. In this framework of geometric ftting, geometric AIC and geometric MDL have been proposed motivated by their statistical counterparts $[6,7]$ and applied to several computer vision problems (e.g., see [6]).

In the context of lens distortion calibration, almost all previous efforts (e.g., [13, 4, 5, 8, 11]) calibrated a £xed, pre-speci£ed distortion model. As such, they may suffer

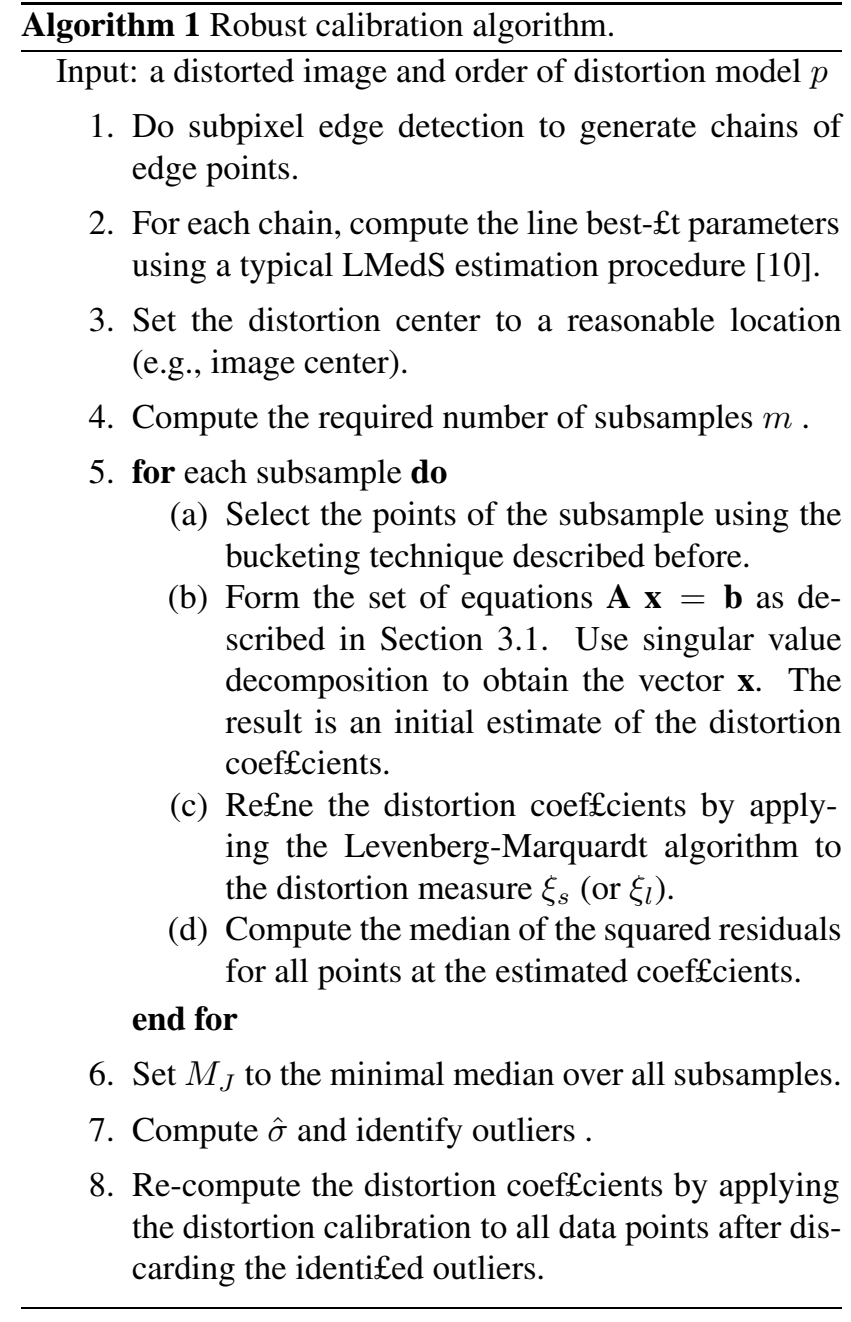

from over/under-parameterization. We are aware of only one work [12] that used a statistical inference method based on Fisher's distribution to identify the proper distortion model. Here we propose to exploit, for the frst time, geometric inference for distortion model selection as it $£$ ts more geometrical problems of computer vision. Since there exists no de£nite criterion to favor any of geometric AIC or geometric MDL [7], we investigate the application of both criteria.

The geometric AIC of a model $S$ is defned as $[6,7]$

$$
\mathrm{G}-\mathrm{AIC}(S)=J(S)+2(\gamma \eta+\mu) \varepsilon^{2},
$$

where $J(S)$ is the residual when data of size $\eta$ are $f t$ to the model $S, \mu$ is the number of degrees of freedom (DOF) of the model, $\gamma$ is the dimension of $S$, and $\varepsilon$ is the noise level in the data. $\gamma$ can also be de£ned as $\gamma=\nu-\rho$, where $\nu$ is the DOF of the observed data and $\rho$ is the co-dimension of the model.

The geometric MDL is similarly de£ned as [7]

$$
\operatorname{G-MDL}(S)=J(S)-(\gamma \eta+\mu) \varepsilon^{2} \log (\varepsilon / R)^{2},
$$


where $R$ is a reference length (we take $R=$ image width).

In order to apply AIC or MDL, we need to know the noise level $\varepsilon$. An unbiased estimator of $\varepsilon$ is obtained from the most general candidate model $S^{0}$ (the one with most DOFs) from (for details, see [7])

$$
\hat{\varepsilon}^{2}=\frac{J\left(S^{0}\right)}{\left(\rho \eta-\mu^{0}\right)},
$$

where $\mu^{0}$ is the DOFs of $S^{0}$. Note that the general model $S^{0}$ contains other candidate models.

For any lens distortion model $S$ with $p$ parameters, GAIC and G-MDL are computed from (3) and (4), respectively, with $\nu=2$ (as each observed point has 2 DOFs), $\rho=1$ (as each observed point has co-dimension of 1), $\gamma=\nu-\rho=1, \mu=2 L+p$ (two for each line in addition to the model parameters), where $\mathrm{L}$ is the number of lines used for distortion calibration, and $\eta$ is the number of points used. The residual $J(S)$ is computed after outlying data have been removed in the robust calibration algorithm. For each candidate distortion model, we compute G-AIC (or G-MDL) and select the model that has the smallest GAIC (or G-MDL), while the noise level is the same for all models and estimated from (5) only from the most general model.

\section{Experimental Results}

In this section, the performance of our technique is assessed using both synthetic and real image data. Because of lack of space, some experiments are reported here, many more can be found in [1].

\subsection{Synthetic Data}

In this experiment, the performance of the proposed approach on model selection is tested and evaluated. A $320 \times 242$ image consisting of 10 lines is used as a test image. The lines were generated with random orientations and positions. We de£ned 3 distortion models: distortionfree model $S_{1}, S_{2}=\left\{K_{1}=20 \times 10^{-6}\right\}$ and, $S^{0}=S_{3}=$ $\left\{K_{1}=20 \times 10^{-6}, K_{2}=30 \times 10^{-9}\right\}$. We carried out a series of simulations as follows. In each time, a distortion model from the defned three is selected at random and points (about 880 in total) sampled from the lines were distorted. To simulate errors in feature extraction, the location of each point was then perturbed in a random direction by a distance governed by a Gaussian distribution with zeromean and standard deviation, $\sigma$, starting from 0.2 pixels up to 1.2 pixels with increment 0.2 .

The proposed calibration approach (skipping the steps needed for the LMedS procedure since no outliers were assumed in this experiment) was applied to these data in order to fnd a proper distortion model and the corresponding distortion parameters. Both the G-AIC and G-MDL criteria were tested, with the noise level estimated using the model $S_{3}$. Moreover, for each value of $\sigma$ the experiment was repeated 200 times, each with a different seed point for the random number generator. Table 1 shows the rate of selecting the correct distortion model at each noise value for both the G-AIC and G-MDL. Clearly G-MDL performs an ex-

Table 1. Rate of identifying the correct distortion model at different noise levels.

\begin{tabular}{|l||c|c|c|c|c|c|}
\hline Criterion & 0.2 & 0.4 & 0.6 & 0.8 & 1.0 & 1.2 \\
\hline G-AIC & $100 \%$ & $91 \%$ & $84 \%$ & $76 \%$ & $68 \%$ & $59 \%$ \\
\hline G-MDL & $100 \%$ & $100 \%$ & $99 \%$ & $93 \%$ & $90 \%$ & $81 \%$ \\
\hline
\end{tabular}

cellent job in selecting the correct model as the rate is no less than $99 \%$ up to noise level=0.6 pixels. Whereas G-AIC performance is considerably less as it tends to favor a model that is one parameter more than the correct model (e.g., $S_{2}$ instead of $S_{1}$ ). This experiment verifes that the proposed approach can indeed select a proper model for lens distortion.

\subsection{Real Data}

We applied the complete, automatic approach to several real images. One such example is the image shown in Fig. 1(a). The acquired image is $640 \times 480$ pixels and typically has noticeable lens distortion due to the cheap wideangle lens. Fig. 1(b) shows the undistorted image using the robust distortion algorithm. Some straight lines are imposed in dashed-lines on the image to help demonstrate the effect of distortion and correction on the image. The approach

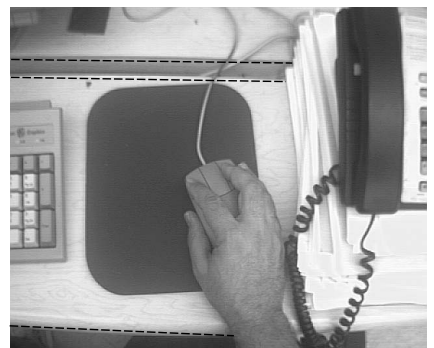

(a)

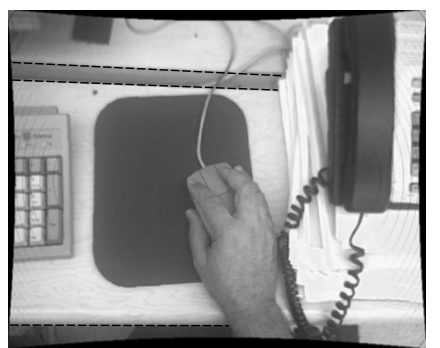

(b)
Figure 1. Performance on desk image (straight lines are imposed on the images to highlight the effect of distortion correction): (a) input distorted image. (b) output undistorted image. 
has been tested with several images with severe lens distortion. One example is shown in Fig. 2(a). The extracted edge chains used in the estimation are shown in Fig. 2(b). It is interesting to see the performance of only the closed-form solutions on this highly-distorted image. The result of the robust algorithm using only the closed-form solution without any non-linear optimization is shown in Fig. 2(c). Surprisingly, the closed-form solution provided a fairly good undistorted image. Afterwards, the non-linear minimization gave the image a $£$ nal polish as seen in Fig. 2(d). The selected distortion model consisted of two radial coeffcients. The approach took $1: 18$ minutes on a SGI-O2 workstation.

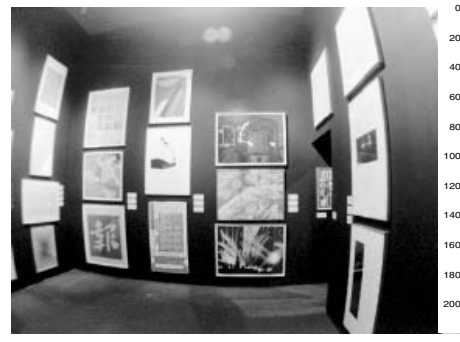

(a)



(c)

(d)

Figure 2. Performance on the exhibit scene: (a) input distorted image (obtained from http://www.media.mit.edu/s̃beck/results/Distortion/), (b) the extracted edge chains, (c) undistorted image using the closed-form solution, (d) £nal undistorted image.

\section{Conclusions}

In this paper, we have proposed a fully automatic approach to non-metric calibration of lens distortion, with some new results in several aspects of the problem. A robust approach to distortion calibration is proposed to discard outliers in the data that might enter the estimation algorithm in different forms. As such, the proposed approach is able to proceed in a fully-automatic manner, whereas almost all existing nonmetric distortion calibration methods need some user involvement for data preparation in one form or another.
The paper has presented fast, closed-form solutions to the distortion coeffcients. This solution serves as an initial point for a following re£ning stage based on non-linear optimization. This represents a major advantage of our approach over the other existing nonmetric calibration techniques.

Moreover, we have proposed a method for distortion model selection based on geometrical inference. We investigated the use of both geometric AIC and MDL. In that regard, we found that G-MDL performed considerably better than G-AIC as the latter seemed to have bias to choose a more general model, the observation that was also made by other researchers in other applications [6].

\section{References}

[1] M. T. Ahmed and A. Farag. Nonmetric calibration of camera lens distortion: Differential methods and robust estimation. IEEE Trans. Image Processing, In Review, 2001.

[2] M. T. Ahmed and A. Farag. Differential methods for nonmetric calibration of camera lens distortion. In Proc. CVPR, Hawaii, Dec. 2001.

[3] H. Akaike. A new look at the statistical model identifcation. IEEE Trans. on Automatic Control, 16(6):716-723, 1974.

[4] D. Brown. Close-range camera calibration. Phtogrammetric Engineering, 37(8):855-866, Aug 1971.

[5] F. Devernay and O. Faugeras. Automatic calibration and removal of distortion from scenes of structured environments. In Proc. SPIE, volume 2567, San Diego, CA, July 1995.

[6] K. Kanatani. Statistical optimization for geometric computation: Theory and Practice. Elsevier Science, 1996.

[7] K. Kanatani. Model selection for geometric inference. Proc. the 5th Asian Conference on Computer Vision, Melbourne, Australia, Jan. 2002.

[8] B. Prescott and G. McLean. Line-based correction of radial lens distortion. Graphical Models and Image Processing, 59(1):39-47, 1997.

[9] J. Rissanen. Universal coding, information, prediction and estimation. IEEE Trans. on Information Theory, 30(4):629636, 1984.

[10] P. Rousseeuw and A. Roy. Robust regression and outlier detection. John Wiley and Sons, 1987.

[11] R. Swaminathan and S. Nayar. Non-metric calibration of wide-angle lenses and polycameras. PAMI, 22(10), Oct. 2000.

[12] G.-Q. Wei, K. Arbter, and G. Hirzinger. Active selfcalibration of robotics eyes and hand-eye relationships with model identifcation. IEEE Transaction Robotics and Automation, 14(1):158-166, Feb. 1998.

[13] J. Weng, P. Cohen, and M. Herniou. Camera calibration with distortion models and accuracy evaluation. PAMI, 14(10), Oct 1992.

[14] Z. Zhang, R. Deriche, O. Faugeras, and Q. Luong. A robust technique for matching two uncalibrated images through the recovery of the unknown epipolar geometry. Artifcial Intelligence Journal, 78:87-119, October 1995. 